\title{
Blocking the Notch signal transduction pathway promotes tumor growth in osteosarcoma by affecting polarization of TAM to M2 phenotype
}

\author{
Shuguang Ren ${ }^{1}$, Xiangmei Zhang ${ }^{1}$, Yueyang $\mathrm{Hu}^{1}$, Jianhua Wu ${ }^{1}$, Yingchao Ju ${ }^{1}, \mathrm{Xu} \mathrm{Sun}^{2}$, Yunjiang Liu ${ }^{1 \wedge}$, \\ Baoen Shan ${ }^{1 \wedge}$ \\ ${ }^{1}$ The Fourth Hospital of Hebei Medical University, Shijiazhuang, China; ${ }^{2}$ Hospital of Stomatology Hebei Medical University, Shijiazhuang, China \\ Contributions: (I) Conception and design: S Ren, Y Liu, B Shan; (II) Administrative support: X Sun, Y Ju; (III) Provision of study materials or patients: \\ J Wu; (IV) Collection and assembly of data: S Ren, X Zhang, Y Hu; (V) Data analysis and interpretation: S Ren, X Zhang, Y Hu; (VI) Manuscript \\ writing: All authors; (VII) Final approval of manuscript: All authors. \\ Correspondence to: Yunjiang Liu; Baoen Shan. The Fourth Hospital of Hebei Medical University, Shijiazhuang, China. \\ Email: lyj818326@outlook.com; baoenshan2016@sina.com.
}

Background: Osteosarcoma is a primary malignant tumor that seriously affects the health and life of patients. It is of great clinical significance to explore the molecular mechanism of osteosarcoma development and develop the corresponding therapeutic targets. Th1/Th2 cytokines in the normal human body are in a state of dynamic balance. When this balance is destroyed, it is related to many diseases such as a tumor, autoimmune disease, microbial infection, transplant rejection, among many others.

Method: The model of mouse tumor-associated macrophage (TAM) was induced by being co-cultured with inducer granulocyte-macrophage colony stimulating factor (GM-CSF) and osteosarcoma S180 cells. The Notch1 knockout mice were obtained by gene targeting technology. The distribution of M1- and M2-type TAMs in the tumor was visualized by immunofluorescence staining. And the western-blot testing was used to detect and quantified the protein level of Notch1 and Th1/Th2-type cytokines.

Results: In this study, the polarization of TAMs to the M2 phenotype occurred after coculture with osteosarcoma S180 cells and secretion level Th1/Th2-type cytokines changed. Also, the expression level of Notch1 reduced significantly. Further, the critical transcription factor Notch1 of the Notch signaling pathway was knocked out in mice. The tumor volume of Notch1 knockout mice was significantly more extensive than of the control mice. The results of microstructural observation on tumor showed that M2type TAMs infiltrated into tumor increased with increased expression of Th2-type cytokines, but M1-type TAMs reduced with reduced expression of Th1-type cytokines.

Conclusions: According to our results, the Notch signal transduction pathway participates in tumor occurrence and growth with a negative role by maintaining Th1/Th2 balance.

Keywords: Notch; tumor-associated macrophage (TAM); Th1/Th2 balance; inhibitory factor; osteosarcoma

Submitted Apr 03, 2020. Accepted for publication Jul 23, 2020.

doi: $10.21037 / \mathrm{atm}-20-3881$

View this article at: http://dx.doi.org/10.21037/atm-20-3881

^ ORCID: Yunjiang Liu: 0000-0001-7202-2004; Baoen Shan: 0000-0002-7877-2524. 


\section{Introduction}

Osteosarcoma is the most common malignant bone tumor in the clinical setting, being the most common in children and teenagers. Osteosarcoma is characterized by local invasion and early lung metastasis with a poor prognosis in patients. The 5 -year survival rate is less than $50 \%$ (1). Unfortunately, the pathogenesis of osteosarcoma is not yet entirely apparent, and the selection of the treatment choice is limited (2).

In recent years, an increasing number of studies have shown that the tumor microenvironment plays a vital role in the development of osteosarcoma, in which tumorassociated macrophages (TAMs) are widely involved in the growth, angiogenesis, metastasis and stem cell-like phenotype of osteosarcoma. A macrophage is a crucial part of the innate immune system with the functions of phagocytosis, antigen presentation, and secretion of various cytokines. It plays a vital role in the inflammation process, defense, repair, and metabolism. In a word, the macrophage is the critical factor for the body to support its stability. Macrophages come from monocytes (3). Bone marrow stem cells differentiate into monocytes, which, after being released into peripheral blood, are further differentiated into tissue-specific macrophages. Then they are induced to polarize into two distinct types, classically activated macrophages (M1 type) and alternatively activated macrophages (M2 type), under the influence of local microenvironment. M1 macrophages are involved in Th1type immune response and promote the inflammation response (4). However, M2 macrophages play a negative role in inflammation response by Th2-type immune response, which may function in the immune escape of tumor cells (5). At present, highly infiltrated M2-type TAMs are discovered in various malignant tumors, including liver cancer, lung cancer, stomach cancer, among many others, which indicates a poor prognosis $(6,7)$.

Macrophage polarization is fine regulated by a variety of genes, molecules, and signal transduction pathways. The notch signaling pathway is a highly conserved signaling pathway that is widely involved in regulating the development and differentiation of tissues, organs, and cells (8). It is noteworthy that there is an enormous amount of research on the Notch signaling pathway regulating macrophage polarization (9).

The study of the regulation mechanism on macrophage polarization can supply innovative ideas for the treatment of related diseases in which macrophages take part. However, at present, the cell typing of TAM in osteosarcoma is not explicit, and there are still differences in its effect on the growth, angiogenesis, and metastasis of osteosarcoma. Therefore, we consider whether the Notch signaling pathway in macrophages participates in the regulation of TAM polarization, which further participates in the development of osteosarcoma. It may provide a way to interfere with macrophage polarization to inhibit tumors. We present the following article in accordance with the ARRIVE reporting checklist (available at http://dx.doi. org/10.21037/atm-20-3881).

\section{Methods}

\section{Ethical compliance}

Experiments were performed under a project license (No.: IACUC- $4^{\text {th }}$ Hos Hebmu-20170117) granted by the institutional ethnics committee of the Fourth Hospital of Hebei Medical University, China, in compliance with this institutional guideline for the care and use of animals.

\section{Induction and identification of the model of mouse TAM cells}

To obtain primary mouse bone marrow cells: Mice were executed by cervical vertebra luxation and sterilized by $75 \%$ ethanol immersion. Then their femurs were removed in clean bench aseptically. The bone marrow cells were washed out by phosphate buffer saline (PBS) with a $5 \mathrm{~mL}$ syringe. The cell suspension was placed in a centrifuge tube and centrifuged at 1,300 rpm for 3 minutes to collect cells. The obtained cells were resuspended by Red Blood Cell Lysis Solution and split at room temperature for 5 minutes. The rest bone marrow cells were resuspended in a $100 \mathrm{~mL} / \mathrm{L}$ fetal bovine serum (FBS)-based Minimum Essential Medium- $\alpha(\alpha M E M)$ medium prepared in advance and inoculated in a $10 \mathrm{~cm}$ dish. The bone marrow cells were cultured for about 16 hours to remove the adherent cells. The non-adherent cells were collected by centrifugation and resuspended with $10 \%$ FBS-based aMEM medium. They were transferred into the dishes or culture plates according to the pre-design. Add the inducer granulocytemacrophage colony stimulating factor (GM-CSF) at a concentration of $40 \mathrm{ng} / \mathrm{mL}$ to stimulate the bone marrow cells to differentiate into monocytes, during which the cell morphology can be observed under a light microscope. Then the induced cells were cultured with osteosarcoma 
S180 cells for 48 hours to obtain TAM cells.

Flow cytometry was used to detect the TAM phenotype. Enzyme linked immunosorbent assay (ELISA) was used to detect the changes in cytokines. Western blot was used to detect the expression of Notch1 protein.

\section{Construction of Notch1 knockout mice}

Two LoxP sequences were inserted into the two ends of the Nothc1 gene in mouse embryonic stem cells by gene targeting technology, which was then implanted into the uterus of pregnant rats. The born mice mated with wild mice, and the F1 mice with Notch1 flox/+ genotype were screened out. They mated with each other, and the F2 mice with Notch1 flox/flox genotype were obtained. Then the Notch1 flox/flox mice mated with Lyz2CRE mice and the F1 mice with Lyz2CRE Notch1 flox/+ genotype mated with each other. The F2 mice with Lyz2Cre Notch1 flox/flox or Lyz2Cre Notch1 flox/+ were selected for later experiments.

\section{The genotyping of transgenic mice}

The DNA of the rat tail was extracted and used for PCR. The reaction systems are as follows: (I) in the first reaction system, the primer sequence is as follows: Primer 1: AGACAAAACAACAAGGTCCAGAAAC; Primer2: GCATCGACCGGTAATGCAGGC. Band size: 465 bp bands were found in the homozygous mice with Notch1 flox/flox genotype, 361 bp bands in the wild mice, and $465 \mathrm{bp}$ and $361 \mathrm{bp}$ bands in the heterozygous mice with Notch 1 flox/+ genotype. (II) In the second reaction system, the primer sequence is as follows: Primer3: AGACAAAACAACAAGGTCCAGAAAC; Primer4: CTGCCAACCATGACAACCTAAGTTC. Band size: $700 \mathrm{bp}$ bands were found in the homozygous mice with Notch1 flox/flox genotype, 600 bp bands in the wild mice, 700 and $600 \mathrm{bp}$ bands in the heterozygous mice with Notch1 flox/+ genotype, and 200 bp bands in the knockout mice with Lyz2CRE Notch1 flox/flox or Lyz2CRE Notch1 flox/+ genotype.

\section{Establishment of a tumor-bearing mouse model}

Five pairs of 6-week-old mice with Lyz2Cre Notch1 f/f and Lyz2Cre Notch1 f/+ genotype from the same source were selected. Osteosarcoma S180 cells suspension, which was in the logarithmic period, was prepared. The above mice were injected with osteosarcoma S180 cells suspension on the right side of their back. After the treatment, the mice were put back into the cage and fed typically. The life status of tumor-bearing mice was observed every day. After 21 days, the tumor was taken out and weighed. And the effect of Notch1 knockout on tumor growth was compared.

\section{Distribution of M1- and M2-type TAMs in the tumor by immunofluorescence staining}

Tumor tissue from five mice separately was removed and put it in $4 \%$ paraformaldehyde overnight. The next day, the tumor tissue was further cut into small pieces and fixed for 12 hours. Then they were dehydrated in a $25 \%$ sucrose solution overnight. After dehydration, they were immersed in a Freeze Embedding Agent (OCT) and quickly placed in liquid nitrogen. The sections were made by freezing microtome and dried on the cationic slide at room temperature. Then they were put into the antigen repair solution, repaired at $80^{\circ} \mathrm{C}$ for 15 minutes, cooled naturally, and sealed for 4 hours. Next, the sections were incubated with the first antibody at $4{ }^{\circ} \mathrm{C}$ overnight, the second antibody at room temperature for 2 hours, the third antibody at room temperature for 40 minutes, and Hoechst Stain solution for 10 minutes in order. After being sealed with $75 \%$ glycerin, the cell morphology tumor was observed by fluorescence microscopy (Olympus BX-51).

\section{Western blot}

Proteins were extracted from the tissue or cells of five mice using Radio Immunoprecipitation Assay (RIPA) lysis buffer (Thermo Fisher Scientific, USA) to collect total protein using five mice separately which was separated on a $12.5 \%$ SDS-PAGE gel and transferred to a polyvinylidene fluoride (PVDF) membrane (Sigma-Aldrich, USA) in turn. The PVDF membrane was blocked by $1 \%$ BSA. Then it was incubated with specific primary antibody made by Bioss Antibodies company (Bioss, China) and then with corresponding secondary antibody (Abcam, UK). Finally, the PVDF membrane was stained using NBT/BCIP Reagent Kit (Thermo Fisher Scientific, USA).

\section{RNA extraction, reverse transcription, and quantitative PCR}

Total RNA was extracted using Trizol (Invitrogen, USA) according to the manufacturer's instructions using five 

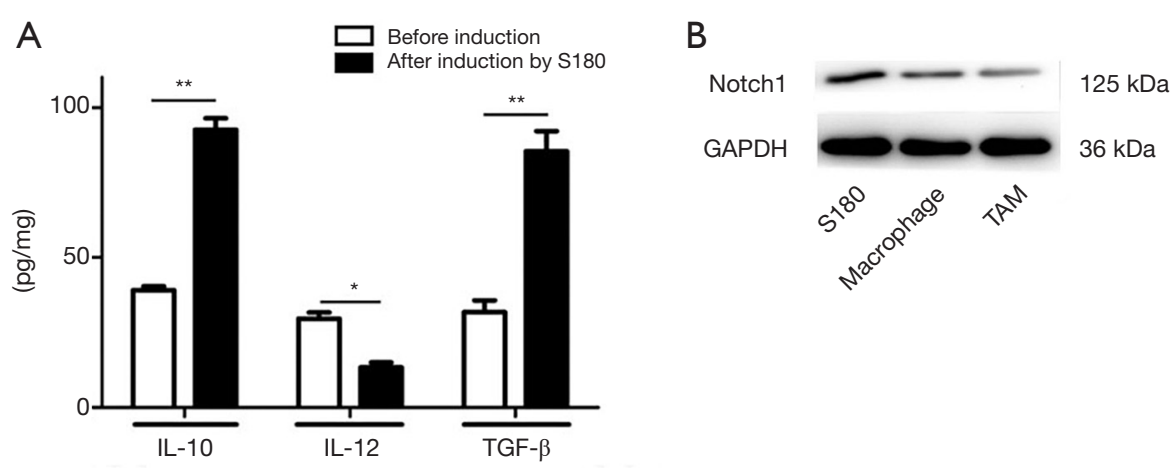

Figure 1 Changes of the expression level of related factors in macrophages after coculture with osteosarcoma S180 cells. (A) The expression level of Th1/Th2-type cytokine was examined by ELISA. The protein level of Th2-type cytokines (IL-10 and TGF- $\beta$ ) increased, and the protein level of Th1-type cytokine (IL-12) decreased. (B) The expression level of Notch1 was detected by western blot. The protein level of Notch1 decreased significantly in macrophages and TAMs after coculturing with osteosarcoma S180 cells.

tissue from five mice separately. First-strand cDNA synthesis was performed using $2 \mu \mathrm{g}$ of total RNA using the Omniscript reverse transcription kit (Qiagen, USA). Quantitative RT-PCR analysis was subsequently performed in triplicate using the QuantiTect SYBR Green PCR system (Qiagen) on a Rotor-Gene 6000 Real-Time PCR Machine (Corbett Research, USA). Data were analyzed using the Rotor-Gene software, which came with the PCR machine. The expression level of GAPDH was used as an internal reference, and expression levels of relevant genes were determined by the $\Delta \Delta C T$ method.

\section{Statistical analysis}

Statistical analysis was performed and the column diagram was generated by GraphPad Prism software using the results from three biological replicates. The statistically significant differences were evaluated by Student's $t$-test ( ${ }^{*} \mathrm{P}$ value $<0.05,{ }^{* *} \mathrm{P}$ value $<0.01,{ }^{* * *} \mathrm{P}$ value $\left.<0.001\right)$.

\section{Results}

\section{Osteosarcoma $S 180$ cells promote polarization of TAM to M2 phenotype}

M1-type and M2-type TAMs secrete Th1-type cytokine (IL-12) and Th2-type cytokines (IL-10, VEGF, TGF- $\beta$, and Arg-1) respectively and play an essential role in the immune response. TAMs were obtained by the co-culture of macrophages and osteosarcoma S180 cells. The type of TAMs was named by examining the related molecular markers by ELISA. As shown in Figure 1A, the secretion of Th1-type cytokines (IL-12) decreased (P value $<0.05$ ), and Th2-type cytokines (IL-10 and TGF- $\beta$ ) increased after induction by osteosarcoma S180 cells, indicating that M2type TAMs increased.

Western blot testing was used to detect the expression of Notch 1 protein, and the content of the Notch 1 protein decreased markedly after their induction by osteosarcoma S180 cells (Figure 1B). These results show that Osteosarcoma S180 cells promote the polarization of the TAMs to the M2 phenotype, and Notch1 may play an essential role in this process.

\section{Knockout of Notch1 promotes tumor growth}

Osteosarcoma S180 cells of $5 \times 10^{6}$ were inoculated subcutaneously in the lower back of the control and experimental mice with Lyz2CRE Notch1 flox/+ or Lyz2CRE Notch1 flox/flox genotype respectively. These mice had consistent growth conditions and weight. After two weeks, the length and width of the tumor were measured with a vernier caliper. Next, the weight was measured with an electronic scale. The tumor volume was calculated according to the formula "Volume $=\mathrm{L} \times \mathrm{S} 2 \times 0.51$ ". The tumor of Notch1 knockout mice was significantly more extensive than that of the control mice (Figure $2 A$ ). The tumor weight of Notch1 knockout mice was about 2.95 times as weight as that of the control mice (9.91 vs. $3.36 \mathrm{~g}$ ), and the tumor volume was about 3.16 times as large as that of the control mice $\left(22.48 v s .7 .10 \mathrm{~cm}^{3}\right)$ (Figure $\left.2 B\right)$. 

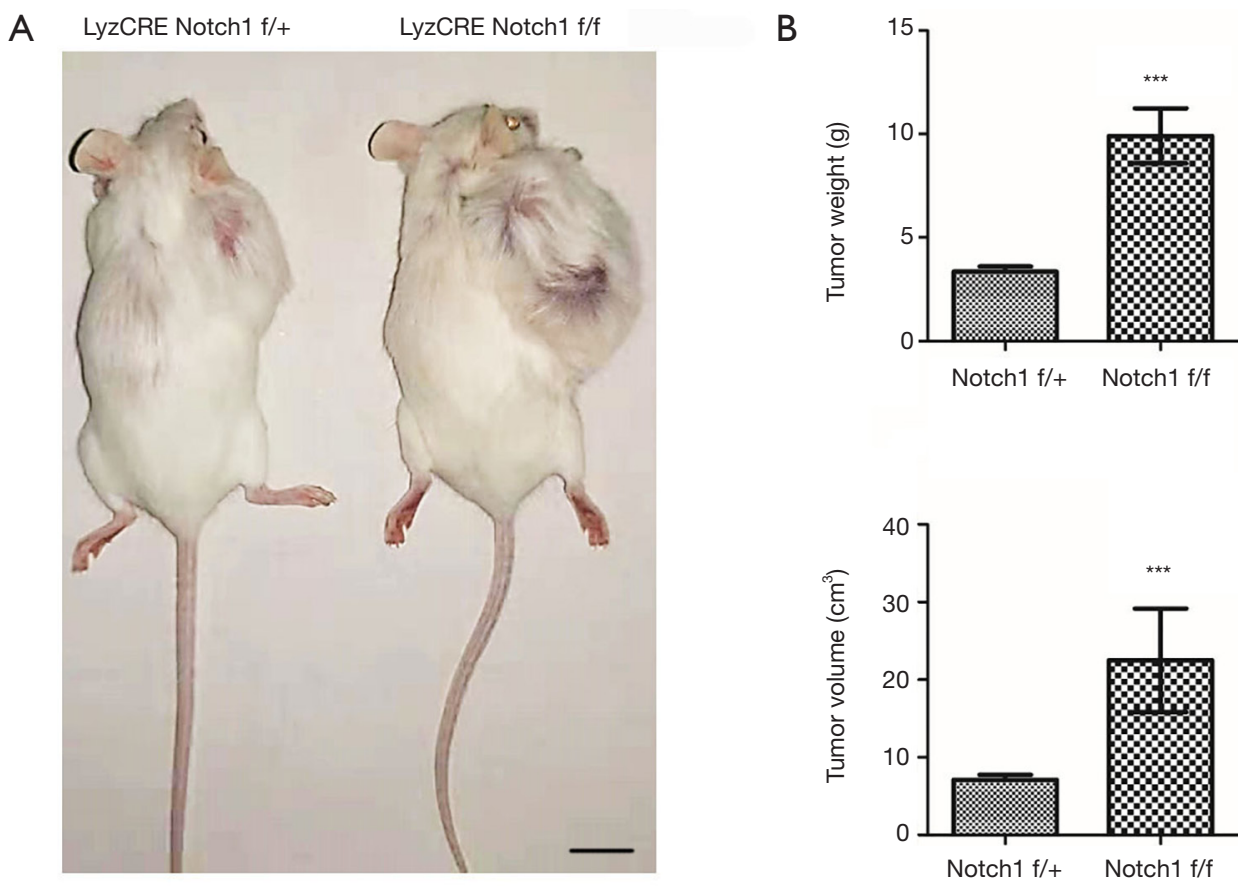

Figure 2 Observation of tumor growth of Nocth1 knockout mice and the control mice (n=10/10). (A) The tumor of Notch1 knockout mice was significantly more extensive than that of the control mice. The black bar indicates $1 \mathrm{~cm}$. (B) The tumor weight and volume of Notch1 knockout mice were $9.91 \mathrm{~g}$ and $22.5 \mathrm{~cm}^{3}$, which were significantly more substantial and more extensive than those of the control mice separately.

\section{Infiltration of M2-type TAMs increased in Notch1 knockout mice}

The tumor tissue was fixed by paraformaldehyde (POM), dehydrated by sucrose, frozen, and embedded in OCT compound and stained by immunofluorescence by turn. iNOS and CD206 are used as cell markers of M1-type and M2-type TAMs, respectively. NPC2, fused with GFP, was used as biomarker of osteosarcoma. The infiltrating M1type and M2-type TAMs in tumor tissue were detected by a fluorescence microscope, and several fields were randomly selected. The results showed that the M1-type TAMs with an expression of iNOS in the osteosarcoma tissue of Notch $1 \mathrm{f} / \mathrm{f}$ mice were significantly less than those in control mice (Figure 3A,B). The M2-type TAMs with an expression of CD206 was significantly more than those in control mice (Figure 3C,D).

\section{Expression of Th2-type cytokines increased in Notch1 knockout mice}

A shift in Th1/Th2 immune balance towards Th2- associated immune responses mays function in the growth and immune escape of tumor cells (10). The above results show that M2-type TAMs increased in tumor tissue of Notch1 knockout mice (Figure 3C,D). M2-type TAMs secrete Th2-type cytokines, which mays be the cause of the more massive tumor in Notch1 knockout mice. Therefore, the expression level of Th1/Th2-type cytokines was detected by western blot and qRT-PCR. The protein and expression level of the Th2-type cytokines (CD206, Arginase-1, IL-10, and TGF- $\beta$ ) was both higher in Notch1 knockout mice than those of the control mice (Figure 4A,B). However, the protein and expression level of the Th1-type cytokines (IL-6, TNF- $\alpha$, and IL-1 $\beta$ ) was both lower in Notch1 knockout mice (Figure 4A,B). These results showed that the block of the Notch signal transduction pathway activated the Th2-type inflammatory reaction, which promotes the tumor growth.

\section{Discussion}

In recent years, many studies have found that TAMs are 

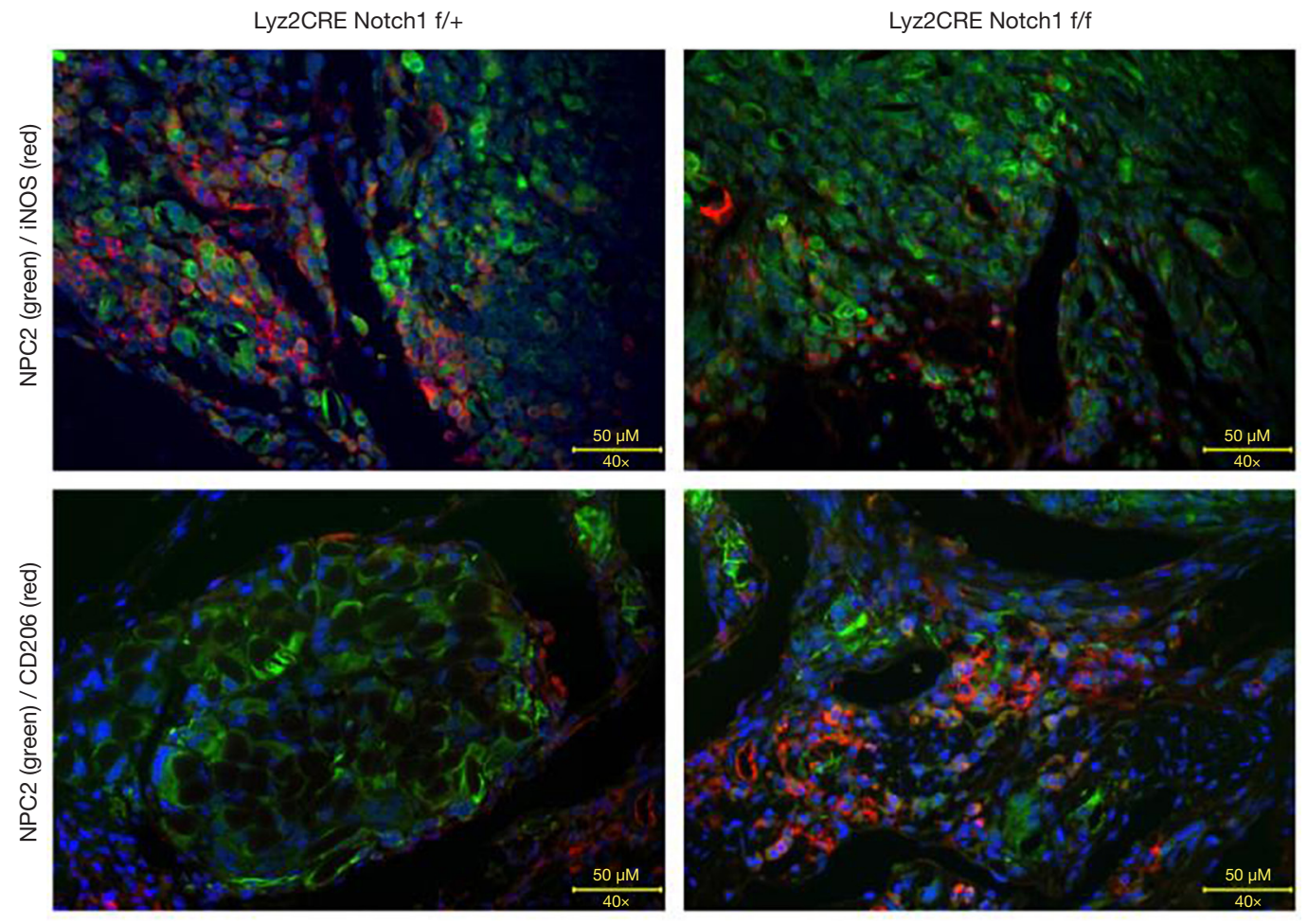

Figure 3 Observation of tumor-associated macrophage (TAM) infiltration by immunofluorescence. M1-type TAMs decreased (A,B) and M2-type TAMs increased (C,D). NPC2, fused with GFP, was used as biomarker of osteosarcoma. The bar is $50 \mu \mathrm{m}$.

closely related to tumor malignancy and poor prognosis, which play a key role in promoting tumor invasion and metastasis (11). In the process of tumor development, TAMs tend to polarize to M2-type TAMs, which are characterized by high-level expression of CD206, CD163, IL-10, low-level expression of IL-2, and secretion of VEGF, TGF- $\beta$, and CCL22 (12). As a result, the Th2-type immune response is induced, which causes immune escape. In this study, monocyte macrophages were demonstrated to be polarized to the M2-type TAMs by being cocultured with osteosarcoma S180 cells. M2-type TAMs secreted less Th1type cytokines and Notch1 protein, but more Th2-type cytokines.

Four Notch receptors (Notch1-4) and five ligands [Deltalike-1, delta-like-3, delta-like-4 (DLL1, 3, 4) and Jagged1, jagged2 (JAG1, 2)] exist in mammals. A Notch receptor is a single transmembrane molecule composed of heterodimers, and its ligand is also a transmembrane molecule (13). Notch signaling pathway functions between cells. Many studies have shown that an abnormal Notch signaling pathway is closely related to macrophage polarization, which plays a significant role in the growth, proliferation, and apoptosis of several tumors $(14,15)$.

In this study, mice with Lyz2Cre Notch $1 \mathrm{f} / \mathrm{f}$ and Lyz2Cre Notch 1 f/+ genotype were obtained when the Notch signaling pathway was blocked. Our results showed that blocking the Notch signal pathway could promote the polarization of the TAM to the M2 phenotype. Further, increased M2-type TAMs decreases the secretion of Th1type cytokines, increases the secretion of Th2 cytokines. In turn, these results increase the Th2-type inflammatory reaction and result in growth and immune escape of tumor cells. As expected, the tumor growth rate of the Notch1 knockout mice was significantly faster than that of the control mice, and the tumor volume was also significantly more extensive.

In conclusion, in the development of tumors, blocking the Notch signaling path promotes the polarization of TAM to the M2 genotype and after promoted the development and metastasis of the tumor. 
A
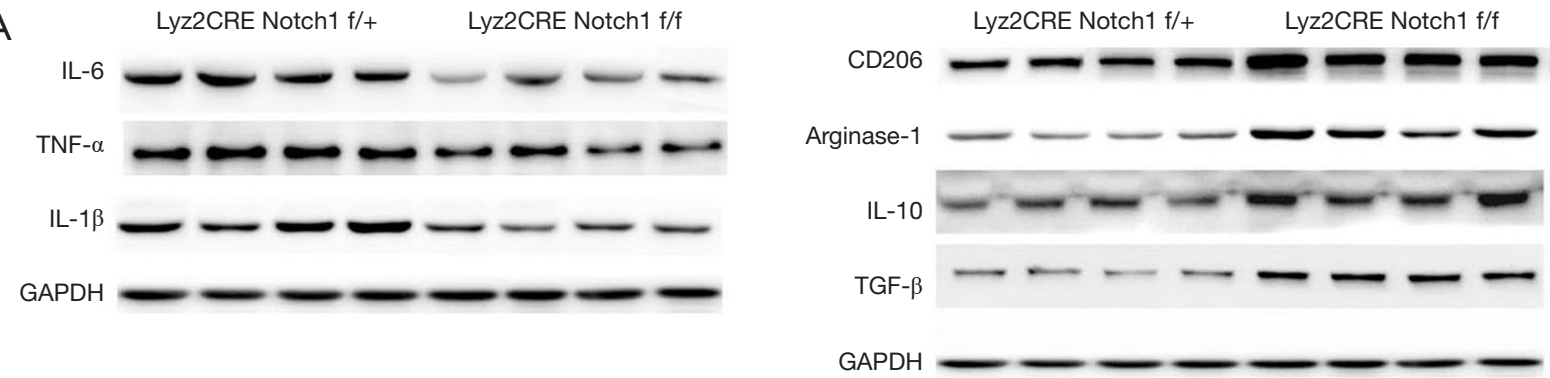

B
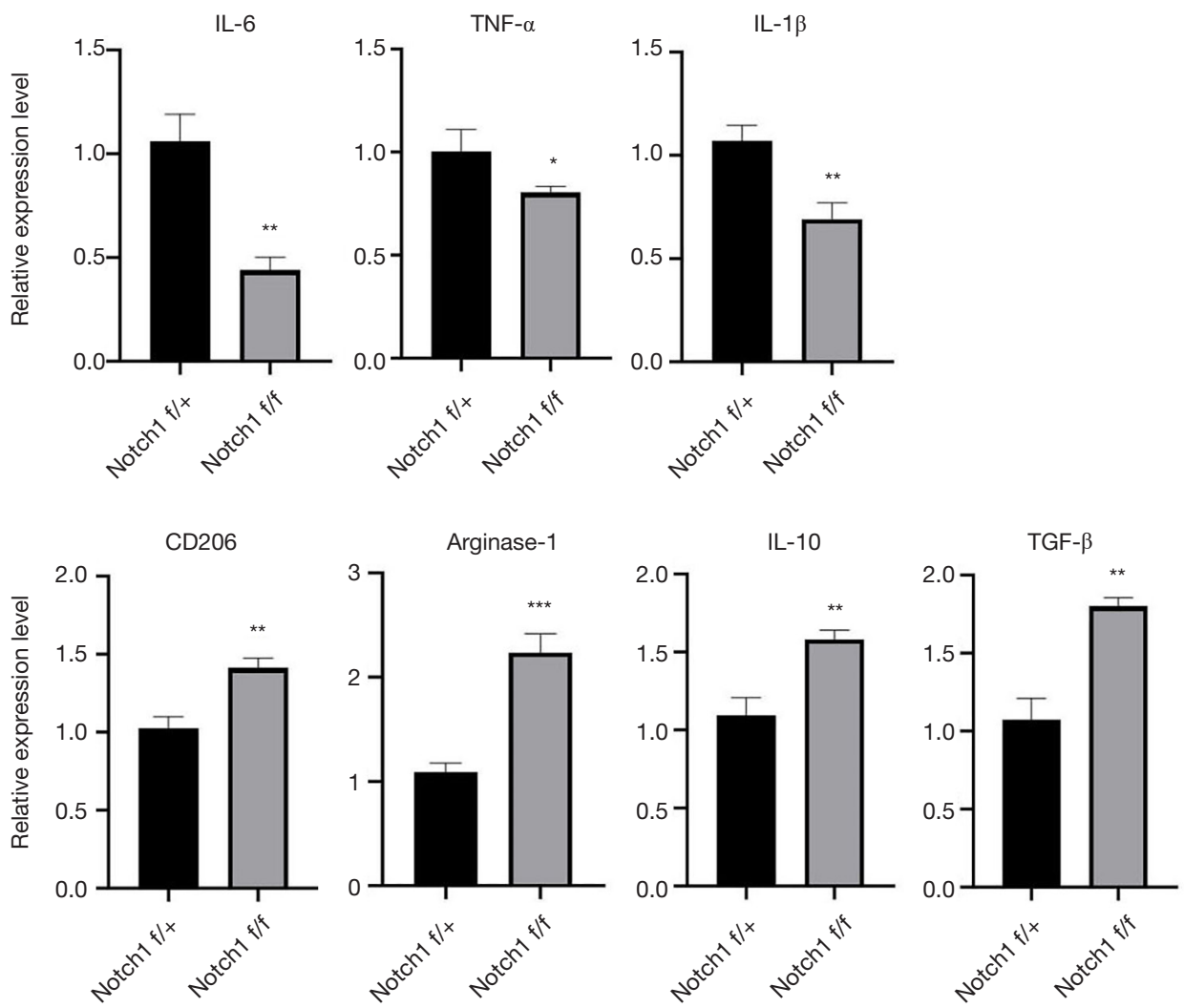

Figure 4 Examine of protein and expression level of Th1/Th2-type cytokines by western blot and qRT-PCR separately. (A) Western blot was used to detect protein levels of Th1/Th2-type cytokines. Th2-type cytokines increased, but Th1-type cytokines decreased in Notch1 knockout mice. (B) qRT-PCR was used to detect the expression level of Th1/Th2-type cytokine genes. Th2-type cytokine gene increased, but the Th1-type cytokine gene decreased in Notch1 knockout mice.

\section{Acknowledgments}

Funding: None.

\section{Footnote}

Reporting Checklist: The authors have completed the ARRIVE reporting checklist. Available at http://dx.doi. org/10.21037/atm-20-3881

Data Sharing Statement: Available at http://dx.doi. org/10.21037/atm-20-3881

Conflicts of Interest: All authors have completed the ICMJE uniform disclosure form (available at http://dx.doi. org/10.21037/atm-20-3881). The authors have no conflicts 
of interest to declare.

Ethical Statement: The authors are accountable for all aspects of the work in ensuring that questions related to the accuracy or integrity of any part of the work are appropriately investigated and resolved. Experiments were performed under a project license (No.: IACUC$4^{\text {th }}$ Hos Hebmu-20170117) granted by the institutional ethnics committee of the Fourth Hospital of Hebei Medical University, China, in compliance with this institutional guideline for the care and use of animals.

Open Access Statement: This is an Open Access article distributed in accordance with the Creative Commons Attribution-NonCommercial-NoDerivs 4.0 International License (CC BY-NC-ND 4.0), which permits the noncommercial replication and distribution of the article with the strict proviso that no changes or edits are made and the original work is properly cited (including links to both the formal publication through the relevant DOI and the license). See: https://creativecommons.org/licenses/by-nc-nd/4.0/.

\section{References}

1. Simpson S, Dunning MD, de Brot S, et al. Comparative review of human and canine osteosarcoma: morphology, epidemiology, prognosis, treatment and genetics. Acta Vet Scand 2017;59:71.

2. An M, Ma WH, Jia HW, et al. Prazosin inhibits the growth and mobility of osteosarcoma cells. Transl Cancer Res 2019;8:1997-2004.

3. Gordon S, Martinez FO. Alternative activation of macrophages: mechanism and functions. Immunity 2010;32:593-604.

4. Verreck FA, de Boer T, Langenberg DM, et al. Human IL-23-producing type 1 macrophages promote but IL-10producing type 2 macrophages subvert immunity to (myco) bacteria. Proc Natl Acad Sci U S A 2004;101:4560-5.

Cite this article as: Ren S, Zhang X, Hu Y, Wu J, Ju Y, Sun X, Liu Y, Shan B. Blocking the Notch signal transduction pathway promotes tumor growth in osteosarcoma by affecting polarization of TAM to M2 phenotype. Ann Transl Med 2020;8(17):1057. doi: 10.21037/atm-20-3881
5. Martinez FO, Helming L, Gordon S. Alternative activation of macrophages: an immunologic functional perspective. Annu Rev Immunol 2009;27:451-83.

6. Shirabe K, Mano Y, Muto J, et al. Role of tumor-associated macrophages in the progression of hepatocellular carcinoma. Surg Today 2012;42:1-7.

7. Farajzadeh Valilou S, Keshavarz-Fathi M, Silvestris N, et al. The role of inflammatory cytokines and tumor associated macrophages (TAMs) in microenvironment of pancreatic cancer. Cytokine Growth Factor Rev 2018;39:46-61.

8. Radtke F, MacDonald HR, Tacchini-Cottier F. Regulation of innate and adaptive immunity by Notch. Nat Rev Immunol 2013;13:427-37.

9. Monsalve E, Pérez MA, Rubio A, et al. Notch-1 upregulation and signaling following macrophage activation modulates gene expression patterns known to affect antigen-presenting capacity and cytotoxic activity. J Immunol 2006;176:5362-73.

10. Lin W, Niu Z, Zhang H, et al. Imbalance of Th1/Th2 and Th17/Treg during the development of uterine cervical cancer. Int J Clin Exp Pathol 2019;12:3604-12.

11. Sica A, Allavena P, Mantovani A. Cancer related inflammation: the macrophage connection. Cancer Lett 2008;267:204-15.

12. Zhang YL, Li Q, Yang XM, et al. SPON2 Promotes M1like Macrophage Recruitment and Inhibits Hepatocellular Carcinoma Metastasis by Distinct Integrin-Rho GTPaseHippo Pathways. Cancer Res 2018;78:2305-17.

13. Louvi A, Artavanis-Tsakonas S. Notch signalling in vertebrate neural development. Nat Rev Neurosci 2006;7:93-102.

14. Fiúza UM, Arias AM. Cell and molecular biology of Notch. J Endocrinol 2007;194:459-74.

15. Kovall RA. Structures of CSL, Notch and Mastermind proteins: piecing together an active transcription complex. Curr Opin Struct Biol 2007;17:117-27. 